\title{
Prevalence and incidence of keratoconus in Norway: a nationwide register study
}

Olav Kristianslund, ${ }^{1,2,3}$ Anne Marie Hagem, ${ }^{1,2}$ Andreas Thorsrud,,${ }^{1}$ Liv Drolsum ${ }^{1,2}$

1 Department of Ophthalmology, Oslo University Hospital, Norway

Address: Mailbox 4956 Nydalen, 0424 Oslo, Norway

2 Institute of Clinical Medicine, University of Oslo, Norway

Address: Mailbox 1171, Blindern, 0318 Oslo, Norway

${ }^{3}$ Corresponding author

Address: Department of Ophthalmology, Oslo University Hospital

Mailbox 4956 Nydalen, 0424 Oslo, Norway

Phone number: +47 22118545

Fax number: +4722119989

Email: olakri@ous-hf.no 


\section{Abstract}

Purpose: To estimate the national prevalence and incidence of keratoconus in Norway

Methods: In this epidemiologic study, data was obtained from the Norwegian Patient Registry, which provides information from all publicly funded specialist care in Norway. Prevalence of keratoconus was estimated from the total number of patients registered with this diagnosis, and incidence from the annual frequency of first-time registrations of patients up to 40 years of age in the period 2010 - 2018. Data on age and gender of the keratoconus patients were also gathered. Results: A total of 9832 keratoconus patients were registered. The estimated prevalence in the general population was 192.1 per 100000 (95\% confidence interval (CI): 188.3 to 195.9), and the estimated annual incidence was 19.8 per 100000 (95\% CI: 18.1 to 21.5). There was a predominance of males (73\%), and mean age for all patients was 37.5 years at first registration in this period.

Conclusion: This study reports the frequency of keratoconus from a national patient register during a time period with available modern diagnostic tools. The estimated prevalence and incidence were higher than most previous estimates and show that keratoconus is not a rare condition. As screening was not part of the study, the true prevalence in the general population may be even higher.

Key words: Keratoconus; Prevalence, Incidence, Corneal collagen crosslinking; CXL; Epidemiology 


\section{Introduction}

Keratoconus is a condition characterized by irregular astigmatism, corneal thinning and development of a cone shape of the cornea (Krachmer et al. 1984). Usually, it develops in adolescence or early adulthood and progresses until the late 20`s or early 30's. Until two decades ago, no effective treatment was available to halt the progression, and corneal transplantation was the only treatment option in patients with far advanced keratoconus. In recent years, corneal collagen crosslinking (CXL) has become the preferred treatment, being highly successful in stopping the progression (Hersh et al. 2011; Hersh et al. 2017; Wollensak et al. 2003). Reports have shown that the introduction of CXL has been followed by a decreased demand for corneal transplantation in this patient group (Godefrooij et al. 2016; Sandvik et al. 2015).

The success of CXL has also led to an increased awareness of the disease, and consequently early referral, sophisticated diagnostics and diligent follow-up. In addition, more knowledge has become available of the risks associated with refractive laser surgery in corneas with signs of keratoconus (Rao et al. 2002). Corneal tomography is now routinely performed prior to such laser procedures (Al-Amri 2018; Elbedewy et al. 2019).

Previous studies of keratoconus have estimated a prevalence in the range of $17.5-86$ per 100 000, and an annual incidence in the range of 1.3 - 5.6 per 100000 (Bak-Nielsen et al. 2019; Hwang et al. 2018; Ihalainen 1986; Kennedy et al. 1986; Lee et al. 2020; Nielsen et al. 2007) with a few studies reporting markedly higher rates (Godefrooij et al. 2017a; Jonas et al. 2009; Millodot et al. 2011; Papali'i-Curtin et al. 2019). This considerable variation is likely related to several factors, including research methodology, populations studied, access to health care and diagnostics (Bak-Nielsen et al. 2019; Godefrooij et al. 2017a). A recent study from The Netherlands using data from an insurance database, reported an annual incidence of 13.3 per 100000 and a prevalence of 265 per 100000 (Godefrooij et al. 2017a). These high estimates indicate that keratoconus may be more common than previous studies have suggested.

Only a few studies on the occurrence of keratoconus in Northern Europe have been reported (Bak-Nielsen et al. 2019; Ihalainen 1986; Nielsen et al. 2007). A recent study from Denmark estimated an annual incidence of 3.6 per 100000 and a prevalence of 44 per 100000 using data from a national patient register (Bak-Nielsen et al. 2019). No studies have reported prevalence or incidence estimates for keratoconus in Norway. The Norwegian public health care system has universal coverage, easy access to good health care and a national patient register that gathers 
information from all publicly funded specialist care. Therefore, we believe Norway is suitable for studying keratoconus epidemiology. Corneal collagen crosslinking was introduced gradually from 2007 in large parts of Norway. Thus, by investigating the most recent years, the data is likely to reflect the era in which modern diagnostic tools and CXL treatment have been available to keratoconus patients. The aim of the present study was to estimate the prevalence and incidence of keratoconus in Norway by investigating the period $2010-2018$.

\section{Materials and methods}

In this epidemiologic study, data was obtained from the Norwegian Patient Registry. This register is administered by the Norwegian Directorate of Health, which also approved use of the data for research purposes. The Data Protection Officer at the research institution waived the need for study approval, as the data were anonymous and on aggregated group level. The research adhered to the Declaration of Helsinki.

The Norwegian Patient Registry gathers information from all publicly funded specialist care in Norway. This includes hospital departments, as well as the vast majority of private practice ophthalmologists since they usually have a public contract. Reporting to the register is mandatory for these groups. Privately financed ophthalmological care, as provided by optometrists and a few private practice ophthalmologists, is not reported to the register. However, we assume that almost all patients with suspected or diagnosed keratoconus that may be eligible for CXL treatment or other types of interventions (e.g. corneal transplantation, intrastromal corneal ring segments or advanced contact lens fitting) are referred to public specialist care. A few privately financed clinics in Norway offer CXL treatment paid by the patient or an insurance company. We have not been able to obtain the exact number; however, this is performed to a very limited extent. In addition, we assume that at least some of these patients have been examined within public specialist care at some point and subsequently been included in the register.

In the present study, we collected data on the number of patients that received the specific International Classification of Disease (ICD)-10 diagnosis H18.6 Keratoconus in the period 2010 - august 2019. Data from 2010 - 2018 was used for calculation of incidence. Information on age and gender was also obtained. A study period from 2010 was chosen because it was the first year with complete diagnostic data in the Norwegian Patient Register. This year was also after the 
introduction of CXL in large parts of Norway. Each patient has a personal identification number in the register, hence we were able to gather information on the number of individuals with a keratoconus diagnosis. This ensured that each patient was only recorded once during the specified time period. Approximately $10 \%$ of the keratoconus registrations were excluded before we received the data. This was due to technical errors (incomplete personal identification numbers, leading to uncertainty whether they were unique patients or additional consultations of already registered patients). Most of these incomplete registrations (87\%) occurred in 2010-2013.

Due to privacy regulations, the register provided aggregated group data without personal identifiable information. Therefore, we could not perform any additional analysis. Furthermore, the register requires a minimum number of cases in each group when reporting anonymous data. Thus, the youngest and oldest age groups for the annual incidence were reported in wider intervals due to few registered keratoconus patients in these ages.

The prevalence of keratoconus was estimated from the total number of registered keratoconus patients in relation to the average number of national inhabitants in the specific time period. The incidence was estimated from the annual number of new registrations of patients up to 40 years of age and the number of national inhabitants in this age group for each specific year. We considered these patients to be the ones at risk of developing keratoconus, and thus the relevant group for estimating incidence, in accordance with others (Godefrooij et al. 2017a). Population numbers were gathered from Statistics Norway (ssb.no). Both the prevalence and the annual incidence are presented per 100 000, with 95\% confidence intervals (CI) calculated in SPSS (version 26, IBM Corp., Armonk, NY, USA).

\section{Results}

A total of 9832 patients were registered with keratoconus in public specialist care in this time period. With an average of 5118664 inhabitants in Norway in the same period, we estimated a prevalence of 192.08 per 100000 (95\% CI: 188.32 to 195.90), corresponding to 1 per 521. Of these patients, $73 \%$ were males and $27 \%$ females. The mean age was 37.5 years at first visit. The age distribution is shown in Table 1. Of these patients with keratoconus, $43 \%$ had their diagnosis registered only in hospital, $23 \%$ in private practice, and $34 \%$ in both. 
The number of new registrations of keratoconus in each year is presented in Figure 1. In the age group up to 40 years, we estimated an annual incidence of 16.33 - 22.95 per 100000 (Figure 2), with a mean of 19.76 per 100000 (95\% CI: 18.13 to 21.49). The gender distribution for new cases is shown in Figure 1 and the age distribution in Figure 3. The highest rate of new cases occurred in the age group $21-30$ years.

\section{Discussion}

Keratoconus has traditionally been considered a quite uncommon condition. However, prevalence estimates have varied (Bak-Nielsen et al. 2019; Hwang et al. 2018; Ihalainen 1986; Kennedy et al. 1986; Lee et al. 2020; Nielsen et al. 2007), and some recent publications have indicated that keratoconus is more common than previously reported (Godefrooij et al. 2017a). The present study used data from a public patient register in Norway, a country with approximately 5.3 million inhabitants. From the obtained data, we estimated a keratoconus prevalence of 192.1 per 100 000, and an annual incidence of 19.8 per 100000 . This is among the highest rates reported from a general population. Since we obtained data from a nationwide mandatory patient register, we believe the present study provides a solid basis for estimating the occurrence of keratoconus. Nevertheless, the numbers are uncertain, and the true prevalence may be even higher.

Most prevalence estimates for keratoconus have been in the range of 17.5 - 86 per 100000 , reported in studies from the US (Kennedy et al. 1986), Finland (Ihalainen 1986), Korea (Hwang et al. 2018; Lee et al. 2020) and Denmark (Bak-Nielsen et al. 2019; Nielsen et al. 2007). However, a few studies in selected age groups have reported significantly higher numbers in studies from the Netherlands (Godefrooij et al. 2017a), India (Jonas et al. 2009), Jerusalem (Millodot et al. 2011) and New Zealand (Papali'i-Curtin et al. 2019). Since it has been suggested that keratoconus is associated with ethnicity (Georgiou et al. 2004; Pearson et al. 2000), one would expect the prevalence to vary globally. Variation in prevalence estimates is likely also related to study designs, completeness of the data, access to health care in various countries, disease criteria and the diagnostics used. It may also be affected by whether the prevalence is estimated for the general population, for population subgroups, or specific age groups, although 
keratoconus is considered a chronic disease. There seems to be a very limited number of nationwide prevalence studies.

In a study from The Netherlands, Godefrooij et al (2017a) estimated an annual incidence of 13.3 per 100000 in the age group 10 - 40 years and a prevalence of 265 per 100000 in the general population, using data from a health insurance database. These estimates are quite similar to ours. Few studies of keratoconus epidemiology from the Nordic countries have been published (Bak-Nielsen et al. 2019; Ihalainen 1986; Nielsen et al. 2007), and none from Norway. Interestingly, a study from Denmark estimated markedly lower rates than ours using data from their national patient register (Bak-Nielsen et al. 2019), although Denmark is close to Norway both in geography and ethnicity. Nevertheless, the Danish register only includes hospital patients. In addition, they did not limit their incidence estimates to include only persons under 40 years of age. Furthermore, the authors suspected their result to be an underestimate, and they reported an increasing incidence over the last decades, in accordance with our findings.

It should be mentioned that some of the keratoconus registrations in the present study, most of them in the first years of the period, were excluded for methodological reasons. This may have resulted in a slight underestimation of the incidence in the first years. On the other hand, an accumulating effect may have occurred from cases diagnosed in the years prior to the study time period. These cases may have contributed to a slight overestimation of the incidence in the first years, and in particular 2010, although this potential bias was partly adjusted for by the register system. We assume that the possible effects of these two factors may offset each other. Nevertheless, if considering 2010 as a washout year, there appeared to be an increasing trend over the time period studied in the present report, although there is some uncertainty in the numbers.

An increase in the number of cases over the years may have several possible explanations. It could be an actual increase in the incidence, possibly related to changes in predisposing factors. However, it seems more likely to be caused by an increased awareness of the disease in parallel with the quite recent introduction of CXL as a successful treatment to stop the progression. Studies from both The Netherlands and Norway have shown a rapid rise in the number of CXL treatments performed in the years just before and after 2010 (Godefrooij et al. 2016; Sandvik et al. 2015). Due to these factors, more patients are probably referred to specialist care for evaluation, as well as followed regularly. Furthermore, new diagnostic tools have been 
introduced and become more easily accessible in many countries in the recent years. This may have increased the number of subclinical patients diagnosed with keratoconus, not least in relation to preoperative evaluation for refractive laser surgery.

An increasing trend may also be a result of immigration from regions with a higher prevalence of keratoconus. Despite the lack of multinational, epidemiologic studies, there seems to be agreement that the prevalence of keratoconus varies by ethnicity. In particular, several studies have indicated a higher prevalence in Asians (in the UK) compared to whites (Cozma et al. 2005; Georgiou et al. 2004; Pearson et al. 2000), although one study reported a lower odds of keratoconus in Asians (in the US) compared to whites, and a higher odds in blacks and latinos (Woodward et al. 2016). Reasons for this inconsistency may be that various populations of Asians have been studied. It has further been discussed whether the differences shown might be related to genetic factors in specific immigration groups (Georgiou et al. 2004). The study from The Netherlands, which found a quite high prevalence of keratoconus in the general population, reported that $22 \%$ of the Dutch population are immigrants (Godefrooij et al. 2017a). In the study from Denmark, they found a lower incidence when immigrants were excluded (Bak-Nielsen et al. 2019). In the present study, we had no information about ethnicity. Approximately $15 \%$ of the population are immigrants or have immigrant parents (Statistics Norway 2019). Furthermore, statistics show that the proportions with an immigrant background have increased in the recent decade especially from Syria, Afghanistan, the Philippines, Thailand, India, China, Somalia, Eritrea and from some European countries. We can only speculate whether this may have affected the incidence in the time period studied.

In the present study, we only included patients coded as H18.6 (ICD-10) and not patients with other corneal ectasias given other diagnosis codes, although it seems likely that some of these have been coded as keratoconus and therefore included. Furthermore, we excluded other corneal conditions, which may have been included to some extent in a few of the previously published reports on keratoconus prevalence (Godefrooij et al. 2017a). Such factors may affect the comparability between studies. Also, age groups/populations studied have varied in previous studies. For example, Jonas et al (2009) reported a high keratoconus prevalence of 2300 per 100000 in Central India in patients above 30 years of age. However, it seems that the only diagnostic criterion in their study was corneal refractive power $>48 \mathrm{D}$, which is not consistent with most other studies. The estimated prevalence became considerably lower when they instead 
used $>49 \mathrm{D}(0.6 \%)$ or $>50 \mathrm{D}(0.1 \%)$. A similarly high keratoconus prevalence was also reported by Millodot et al (2011) in Jerusalem, however, this study was conducted in a college student population rather than a general population study.

The Norwegian Patient Registry is nationwide and mandatory. Therefore, we believe that the presented results actually reflect the frequency of keratoconus patients examined within public specialist care in the specified time period. Our estimates clearly indicate that the prevalence in Norway is higher than most previous reports globally, but in accordance with the recent study from The Netherlands, although this study calculated the prevalence from the incidence estimate (Godefrooij et al. 2017a). Still, it seems likely that even our study underestimates the true prevalence of keratoconus, at least in higher age groups. We assume that most keratoconus patients have at least one consultation within public specialist care around the time of diagnosis, in particular if they are in the age range considered candidates for CXL treatment. We therefore hypothesize that the prevalence in the age group 21-30 years, of 409 per 100000 , is near a true estimate. In higher ages, however, we suspect that some of the patients diagnosed in privately financed health care were not referred to public specialist care if not considered candidates for treatment (e.g. patients above 40 years with stable refraction). Such patients will not be included in the register. Also, some older patients with previously diagnosed and stable disease may not have been included in the specified time period. Reasons for this may be non-regular examinations or follow-up by an optometrist within private care. These assumptions are supported by the age distribution in the present study which shows a lower prevalence in higher age groups; a finding that is not consistent with the chronicity of the disease. Factors contributing to an overestimation of keratoconus may also have been present, related to lack of standardized diagnosis criteria and varied distribution of corneal tomographs among ophthalmologists in Norway. If a patient suspected of having keratoconus is (wrongly) coded with this diagnosis and referred to a hospital department, which then concludes with regular astigmatism, the patient will contribute to an overestimation of keratoconus due to the first diagnosis registration. We believe these cases are few, but may represent an uncertainty in the data. Altogether $77 \%$ of keratoconus cases had their diagnosis registered in the hospital setting.

An implication from our findings is nevertheless that keratoconus should not be considered a rare condition, neither by clinicians, nor by policy makers that are allocating health care resources. It has previously been shown that CXL is a cost effective treatment in these patients 
(Godefrooij et al. 2017b). Efforts should be made to diagnose keratoconus at an early stage to be able to consider treatment before vision is considerably reduced.

A screening study would reveal a more accurate prevalence of keratoconus. However, we are not aware of any large screening studies from a general population. Two previous studies of patients seeking surgery for refractive errors in Saudi Arabia (Al-Amri 2018) and the Egyptian delta (Elbedewy et al. 2019) found that the proportions with keratoconus or keratoconus suspect were $32.3 \%$ and $1.1 \%$, respectively. Although these are highly selected patient populations, and the results showed great variation, it indicates a considerable amount of undiagnosed keratoconus in the population.

The highest number of newly registered keratoconus patients in the present study was found in the age group 21-30 years, followed by the next two decades. A peak age interval in the 20's seems to be in accordance with previous studies and in the age range in which CXL treatment is usually performed. However, we suspect that the age distribution was otherwise affected by methodological limitations. Since we were only able to obtain register data from 2010, it means that a number of previously diagnosed keratoconus patients have been registered as new cases if they had at least one consultation in public specialist care within the time period of the study. It seems reasonable to assume that this effect tends to overestimate both the incidence in higher age groups and the mean age at diagnosis. On the other hand, some older patients were probably diagnosed in privately financed health care and were not referred to public specialist care, thus excluding them from the statistic. To adjust for some of these aspects, incidence was calculated by including only patients up to 40 years, in accordance with others (Godefrooij et al. 2017a). Still, there is considerable uncertainty related to the incidence estimates in the present study. We therefore believe these numbers should be interpreted with caution.

In the present study, we found a male predominance of $73.4 \%$. The same trend has been shown in some previous publications (Fink et al. 2005; Godefrooij et al. 2017a; Ihalainen 1986; Millodot et al. 2011; Pearson et al. 2000), although others have reported no gender difference (Hwang et al. 2018; Kennedy et al. 1986), or a higher proportion of females (Assiri et al. 2005; Jonas et al. 2009). There are to date few explanations for the possible gender difference in keratoconus, although hormonal factors have been hypothesized (Gordon-Shaag et al. 2015). Furthermore, the causes of the inconsistency in previously reported gender distributions are uncertain. One may hypothesize that the mean age of first-time pregnancy in a population may 
affect the rate of keratoconus in females, since pregnancy is considered a risk factor for keratoconus progression (Bilgihan et al. 2011; Naderan \& Jahanrad 2017). However, further studies are needed to evaluate these aspects.

The strength of the present study is that data was obtained from a mandatory national health register. Keratoconus has a specific ICD-10 code and it seems likely that the correct number of registered patients has been reported, except for some uncertainty related to the $10 \%$ excluded registrations. Limitations are the lack of information from privately financed health care, and furthermore that we have not performed any screening of healthy individuals. A true national prevalence and incidence could therefore not be estimated. In addition, the diagnosis of keratoconus was not standardized, as the coding has been conducted by several clinicians and not according to a strict study protocol. This also includes some potential inconsistency in the coding of other corneal ectasias such as post-LASIK ectasia. Furthermore, the availability of corneal tomographs varies. A quite short study period also provides some uncertainty, in particular for the incidence estimates, as known keratoconus cases prior to 2010 may have been counted as new cases. However, we were able to estimate incidence for several consecutive years, and the data included the years after introduction of modern diagnostics and CXL, which we believe are strengths of this study.

In conclusion, we found estimates of keratoconus prevalence and incidence higher than in most previous reports. This may in part be explained by the increased awareness of the disease combined with more available diagnostics in recent years. That keratoconus seems to be more common than previously suspected is important knowledge for clinicians, as well as policy makers and health economists. A male predominance is likely, although the gender distribution has varied in previous reports. A multinational study screening healthy individuals could provide more answers regarding both the true prevalence and the possible variation with ethnicity and gender. 


\section{Acknowledgement:}

Funding/support: This study has received no financial support

Conflict of interest: The authors have no conflicts of interest related to this work

Disclaimer: Information from the Norwegian Patient Registry has been used in this publication. The interpretation and reporting of these data are the sole responsibility of the authors, and no endorsement by the Norwegian Patient Registry is intended nor should be inferred. 


\section{References}

Al-Amri AM (2018): Prevalence of Keratoconus in a Refractive Surgery Population. J Ophthalmol 2018: 5983530.

Assiri AA, Yousuf BI, Quantock AJ \& Murphy PJ (2005): Incidence and severity of keratoconus in Asir province, Saudi Arabia. Br J Ophthalmol 89: 1403-1406.

Bak-Nielsen S, Ramlau-Hansen CH, Ivarsen A, Plana-Ripoll O \& Hjortdal J (2019): Incidence and prevalence of keratoconus in Denmark - an update. Acta Ophthalmol 97: 752-755.

Bilgihan K, Hondur A, Sul S \& Ozturk S (2011): Pregnancy-induced progression of keratoconus. Cornea 30: 991-994.

Cozma I, Atherley C \& James NJ (2005): Influence of ethnic origin on the incidence of keratoconus and associated atopic disease in Asian and white patients. Eye (Lond) 19: 924-925; author reply 925-926.

Elbedewy HA, Wasfy TE, Soliman SS, Sabry MM, Awara AM, El Emam SY, Shafik HM \& Alam MR (2019): Prevalence and topographical characteristics of keratoconus in patients with refractive errors in the Egyptian delta. Int Ophthalmol 39: 1459-1465.

Fink BA, Wagner H, Steger-May K, Rosenstiel C, Roediger T, McMahon TT, Gordon MO \& Zadnik $\mathrm{K}$ (2005): Differences in keratoconus as a function of gender. Am J Ophthalmol 140: 459468.

Georgiou T, Funnell CL, Cassels-Brown A \& O'Conor R (2004): Influence of ethnic origin on the incidence of keratoconus and associated atopic disease in Asians and white patients. Eye (Lond) 18: 379-383.

Godefrooij DA, de Wit GA, Uiterwaal CS, Imhof SM \& Wisse RP (2017a): Age-specific Incidence and Prevalence of Keratoconus: A Nationwide Registration Study. Am J Ophthalmol 175: 169-172.

Godefrooij DA, Gans R, Imhof SM \& Wisse RP (2016): Nationwide reduction in the number of corneal transplantations for keratoconus following the implementation of cross-linking. Acta Ophthalmol 94: 675-678.

Godefrooij DA, Mangen MJ, Chan E, O'Brart DPS, Imhof SM, de Wit GA \& Wisse RPL (2017b): Cost-Effectiveness Analysis of Corneal Collagen Crosslinking for Progressive Keratoconus. Ophthalmology 124: 1485-1495.

Gordon-Shaag A, Millodot M, Shneor E \& Liu Y (2015): The genetic and environmental factors for keratoconus. Biomed Res Int 2015: 795738.

Hersh PS, Greenstein SA \& Fry KL (2011): Corneal collagen crosslinking for keratoconus and corneal ectasia: One-year results. J Cataract Refract Surg 37: 149-160.

Hersh PS, Stulting RD, Muller D, Durrie DS, Rajpal RK \& United States Crosslinking Study G (2017): United States Multicenter Clinical Trial of Corneal Collagen Crosslinking for Keratoconus Treatment. Ophthalmology 124: 1259-1270.

Hwang S, Lim DH \& Chung TY (2018): Prevalence and Incidence of Keratoconus in South Korea: A Nationwide Population-based Study. Am J Ophthalmol 192: 56-64.

Ihalainen A (1986): Clinical and epidemiological features of keratoconus genetic and external factors in the pathogenesis of the disease. Acta Ophthalmol Suppl 178: 1-64. 
Jonas JB, Nangia V, Matin A, Kulkarni M \& Bhojwani K (2009): Prevalence and associations of keratoconus in rural maharashtra in central India: the central India eye and medical study. Am J Ophthalmol 148: 760-765.

Kennedy RH, Bourne WM \& Dyer JA (1986): A 48-year clinical and epidemiologic study of keratoconus. Am J Ophthalmol 101: 267-273.

Krachmer JH, Feder RS \& Belin MW (1984): Keratoconus and related noninflammatory corneal thinning disorders. Surv Ophthalmol 28: 293-322.

Lee HK, Jung EH \& Cho BJ (2020): Epidemiological Association Between Systemic Diseases and Keratoconus in a Korean Population: A 10-Year Nationwide Cohort Study. Cornea 39: 348-353.

Millodot M, Shneor E, Albou S, Atlani E \& Gordon-Shaag A (2011): Prevalence and associated factors of keratoconus in Jerusalem: a cross-sectional study. Ophthalmic Epidemiol 18: 91-97.

Naderan M \& Jahanrad A (2017): Topographic, tomographic and biomechanical corneal changes during pregnancy in patients with keratoconus: a cohort study. Acta Ophthalmol 95: e291-e296.

Nielsen K, Hjortdal J, Aagaard Nohr E \& Ehlers N (2007): Incidence and prevalence of keratoconus in Denmark. Acta Ophthalmol Scand 85: 890-892.

Papali'i-Curtin AT, Cox R, Ma T, Woods L, Covello A \& Hall RC (2019): Keratoconus Prevalence Among High School Students in New Zealand. Cornea 38: 1382-1389.

Pearson AR, Soneji B, Sarvananthan N \& Sandford-Smith JH (2000): Does ethnic origin influence the incidence or severity of keratoconus? Eye (Lond) 14 ( Pt 4): 625-628.

Rao SN, Raviv T, Majmudar PA \& Epstein RJ (2002): Role of Orbscan II in screening keratoconus suspects before refractive corneal surgery. Ophthalmology 109: 1642-1646.

Sandvik GF, Thorsrud A, Raen M, Ostern AE, Saethre M \& Drolsum L (2015): Does Corneal Collagen Cross-linking Reduce the Need for Keratoplasties in Patients With Keratoconus? Cornea 34: 991-995.

Statistics Norway. Retrieved from https://www.ssb.no/ Accessed June 2020.

Wollensak G, Spoerl E \& Seiler T (2003): Riboflavin/ultraviolet-a-induced collagen crosslinking for the treatment of keratoconus. Am J Ophthalmol 135: 620-627.

Woodward MA, Blachley TS \& Stein JD (2016): The Association Between Sociodemographic Factors, Common Systemic Diseases, and Keratoconus: An Analysis of a Nationwide Heath Care Claims Database. Ophthalmology 123: 457-465 e452. 


\section{Figure legends}

Figure 1 Number of first-time registrations of keratoconus in public health care in Norway 2010 - 2018; reported for all patients (solid line), males (dotted line) and females (dashed line). Data were from the Norwegian Patient Registry. Patients diagnosed with keratoconus prior to 2010 were included in these numbers if registered with the diagnosis code in the study period.

Figure 2 Estimated annual incidence of keratoconus in Norway in the period 2010 2018, per 100000 in the age group 0 - 40 years. Error bars represent the 95\% confidence interval. Incidence was estimated from first time registrations of the diagnosis in the Norwegian Patient Registry. Patients diagnosed with keratoconus prior to 2010 were included in these numbers if registered with the diagnosis code in the study period.

Figure 3 Estimated annual incidence of keratoconus in different age groups in Norway, reported as the average annual incidence in the years 2010 - 2018, per 100000 . Error bars represent the $95 \%$ confidence interval. Incidence was estimated from first time registrations of the diagnosis in the Norwegian Patient Registry. Patients diagnosed with keratoconus prior to 2010 were included in these numbers if registered with the diagnosis code in the study period, thus, an unknown proportion was not newly diagnosed keratoconus. 